\title{
Monitoring fine-scale vegetation cover change using UAV-mounted multispectral cameras: A practical approach
}

Justin James van Blerk ( $\sim$ jvanblerk@gmail.com )

University of Cape Town https://orcid.org/0000-0003-1626-3270

Adam West

University of Cape Town

Julian Smit

University of Cape Town

Res Altwegg

University of Cape Town

Michael Timm Hoffman

University of Cape Town

\section{Research Article}

Keywords: UAV, pseudoinvariant features, NDVI, vegetation, area cover, multispectral, radiometric normalisation, remote sensing; post-fire; shrublands

Posted Date: February 22nd, 2022

DOI: https://doi.org/10.21203/rs.3.rs-1337718/v1

License: (c) (i) This work is licensed under a Creative Commons Attribution 4.0 International License. Read Full License 
1 Monitoring fine-scale vegetation cover change using UAV-mounted multispectral cameras: A practical

2 approach

3 J. J. van Blerk a (Author for correspondence, jvanblerk@ gmail.com, +27 83271 4215),

4 A. G. West $a$, J. Smit ${ }_{b}$, R. Altwegg $c$, M. T. Hoffman ${ }_{d}$

5

6 a Department of Biological Sciences, University of Cape Town, Private Bag X3, Rondebosch 7701, South Africa

7 b Department of Geomatics, University of Cape Town, Private Bad X3, Rondebosch 7701, South Africa

8 c Centre for Statistics in Ecology, Environment and Conservation, Department of Statistical Sciences, University

9 of Cape Town, Private Bag X3, Rondebosch 7701, South Africa

10 dPlant Conservation Unit, Department of Biological Sciences, University of Cape Town, Private Bag X3,

11 Rondebosch 7701, South Africa

12

13 ORCID ID's:

14 J.J.vanBlerk: 0000-0003-1626-3270

15 A.G.West: 0000-0002-9352-9282

16 M.T.Hoffman: 0000-0002-5843-2397

17 R.Atwegg: 0000-0002-4083-6561

18 J.Smit: 0000-0001-8664-8059

19

20 
1. Monitoring fine-scale vegetation processes over wider spatial extents is often logistically challenging.

2. We demonstrate a practical approach for monitoring high-resolution (i.e sub-meter) vegetation cover change using UAV-mounted multispectral cameras. We use the post-fire shrublands of the Cape Floristic Region, South Africa as a case study.

3. Repeated NDVI images of post-fire sites were generated biannually over three years using UAVmounted multispectral cameras and commercial, image-processing software. We applied a procedure for identifying and correcting temporal radiometric noise in repeated NDVI images using pseudoinvariant features (PIFs). We then extracted vegetation cover data using NDVI thresholding. The quality of UAV-based vegetation cover data was assessed using detailed ground-truthing.

4. Relative radiometric image normalization reduced radiometric noise in NDVI data and resulted in stronger correlations between UAV-based and ground-based area cover measurements over time.

5. UAV-mounted multispectral cameras are effective high-resolution vegetation monitoring tools which could help ecologists to investigate fine-scale vegetation processes across wider spatial extents.

Keywords: UAV, pseudoinvariant features, NDVI, vegetation, area cover, multispectral, radiometric normalisation, remote sensing; post-fire; shrublands 
48

49

50

51

52

53

54

55

56

57

58

59

60

61

62

63

64

65

66

67

68

69

Monitoring changes in vegetation cover is central to understanding dynamic ecosystems. Changes in vegetation cover could relate to a variety of important vegetation processes including post-disturbance recovery (Veraverbeke et al., 2012; Gitas et al., 2013), succession, ecosystem degradation or land use change (Cammeraat et al., 2005), growth seasonality (Shoshany et al., 1995) or responses to climate change (Port et al., 2012). The recent introduction of affordable unmanned aerial vehicles (UAVs) and lightweight multispectral cameras has promised to deliver unprecedented accuracy of land cover change, yet there remain significant challenges to overcome as ecologists adopt this technology (Rasmussen et al., 2016).

Typically, studies monitoring vegetation change have suffered a trade-off between scale and resolution. For example, fine-scale physiological or demographic studies (West et al., 2012; Esler et al., 2015; van Blerk et al., $2021 \mathrm{a}, \mathrm{b})$ are often carried out over a relatively small area or conducted using a small sample of target species. The detailed, high-effort nature of such measurements can provide a mechanistic understanding of vegetation processes but also limits the spatial coverage which is logistically possible in the study. On the other end of the scale, regional or landscape-level patterns in vegetation are generally investigated through aerial or satellite imagery (Cramer \& Hoffman, 2015; Slingsby et al., 2020). While this type of imagery makes it possible to study broad patterns over vast regions of the Earth's surface, the information generated from these studies is only as detailed as the resolution and detail of the available imagery. Generally, satellite imagery is too coarse to detect individual plant responses (Aplin, 2005), thus limiting the effectiveness of this data in studies which require the detection of very fine-scale vegetation processes. Notwithstanding, vegetation studies have attempted to bridge the gap between fine-scale and broad-scale resolution by employing methods of estimation for assessing vegetation at intermediate scales. This includes using measures such as 'area cover' or 'canopy density'. Unfortunately, many of these methods of assessing vegetation at intermediate scales can be problematic. For example, visual estimates of area cover tend to be subjective and are difficult to keep consistent over a study period (Bergstedt et al., 2009).

UAVs offer the possibility of monitoring fine-scale vegetation processes over larger spatial scales, essentially bridging the gap between fine-scale and regional level studies. Many important vegetation processes (e.g. shrub resprouting after fire) occur at the sub meter resolution and thus require finer resolution monitoring tools for their detection. This technology is currently being applied to high precision agriculture (Zhang \& Kovacs, 2012; Duveiller \& Defourny, 2010; Marcial-Pablo et al. 2019) and is becoming more commonly used in a 
wide range of ecological studies (e.g. van Iersel et al., 2018; Estrany et al. 2019; Sotille et al., 2020; Slingsby et al., 2020). Quantifying vegetation cover change using repeated imagery can be effective using vegetation indices which are representative of some ecological or biophysical process (Aplin, 2005). The normalized difference vegetation index (NDVI) (Tucker, 1979) is perhaps the most commonly used vegetation index in remote sensing, providing an indication of photosynthetic activity. NDVI makes use of the high absorption of red light in leaf material and a high reflectance of near infrared (NIR) light to differentiate plants from nonphotosynthetic materials within an image. This phenomenon can be useful for accurately computing high resolution vegetation area cover from NDVI images (Ghazal et al., 2015; Marcial-Pablo et al. 2019).

Tracking high-resolution environmental patterns in time-series data is challenging if measurement-related inconsistencies occur within the time-series (Bao et al., 2012; Zhou et al., 2016). This can result from human subjectivity in measurement, or calibration issues related to measurement instruments. Such measurementinduced noise should be identified and normalised before any meaningful environmental patterns can be extracted from the data. This is especially true if measurement-related variation in the data is larger than the variation associated with the process being observed. In remote sensing, temporal radiometric image distortion is common in time-series and thus normalisation is often required before any interpretations of imagery data can be made. Therefore, the general uptake of UAV-based monitoring technology by many environmental scientists needs to be matched by the development of appropriate methodological protocols. The availability of affordable monitoring equipment is still a relatively new phenomenon and thus there are few critical assessments of the utility of these tools for general use in research.

Here we documented the ability of commercial multispectral cameras mounted on UAVs to reliably track highresolution vegetation area cover changes during a three-year, post-fire recovery period in the Cape Floristic Region, South Africa. Using our data as an example, we propose a practical approach to conducting repeatable vegetation cover change detection for non-remote sensing specialists: 1) assessing the temporal radiometric stability of repeated NDVI images, based on trackable, pseudo invariant features (PIFs), 2) applying relative radiometric normalization by matching PIFs between uncorrected and reference images and 3) using thresholding to isolate vegetation pixels within NDVI images and compute high-resolution changes in vegetation cover over time. The effectiveness of our approach was quantified by comparing remotely sensed vegetation cover estimates with detailed ground area cover measurements that were recorded simultaneously to UAV image surveys. 


\subsection{Study site}

107 We monitored post-fire vegetation recovery over three years at the Drie Kuilen Nature Reserve $\left(33.5805^{\circ} \mathrm{S}\right.$, $\left.20.0332^{\circ} \mathrm{E}\right)$ in the Western Cape of South Africa. The vegetation of this region comprises of the fire-prone, Matjiesfontein Shale Renosterveld and Langeberg Sandstone Fynbos (Mucina \& Rutherfoord, 2006). The region is semi-arid with $<300 \mathrm{~mm}$ mean annual rainfall. We conducted our study in a Fynbos and a Renosterveld site located within a kilometre of each other (with $<200 \mathrm{~m}$ altitude difference), either side of a distinct ecotone. We monitored 12 permanent $16 \mathrm{~m}^{2}$ plots within each site (i.e. Fynbos $\times 12$ and Renosterveld $\times$ 12) after a controlled burn in May 2016. Controlled burns cleared plots of all above ground biomass (van Blerk et al., 2021 a).

\subsection{Multispectral imagery collection using UAVs:}

116 Starting in February 2017, aerial imagery of Fynbos and Renosterveld sites was captured twice a year using

117 UAVs (DJI Phantom 3 Professional - https://www.dji.com) and a mounted multispectral camera (Parrot sequoia 118 - https://www.parrot.com). The Parrot sequoia was mounted onto the DJI Phantom 3 Professional with a Parrot 119 Sequoia Mount Kit. Unfortunately, the camera mount did not include a gyro meaning that the camera position was fixed relative to the UAV. This meant that care had to be taken to keep the camera positioning on the UAV constant over repeated surveys. On each survey date; hundreds of individual images, with $>80 \%$ overlap, were captured from a height of $15-20 \mathrm{~m}$ during a 2D grid flight path. Survey flight paths were programmed using AtlasFlight (https://micasense.com) software. The Parrot sequoia captured red (640-680nm) and near infrared (NIR) (770-810nm) bands using multispectral sensors and also captured RBG (Red, Green, Blue) images. Repeated UAV surveys were always conducted as close to midday as possible to reduce shadows around vegetation. Flight times did not exceed 25 minutes due to battery-life limitations. UAV surveys were also conducted on days with $0 \%$ cloud cover.

Single-band red and NIR images were calibrated using standard Pix4D Mapper (https://www.pix4d.com) software calibrations. These included i) camera corrections (e.g. vignetting, ISO, dark current etc.), ii) radiometric calibration panel reflectance values and iii) sunshine sensor data. Immediately before and after surveys, images of a MicaSense calibrated reflectance panel (https://support.micasense.com) and 18\% gray cards were captured (Fig. 1). This allowed for the Pix4D Mapper image-processing software to apply 
radiometric calibrations to single band images (e.g. red and NIR) via the empirical line method before generating multi-band vegetation indices (e.g. NDVI). The Pix4D software used only a single radiometric target to perform image corrections but the other target served as a backup in case of calibration images being overexposed. Additionally, a Sunshine Sensor module (https://www.parrot.com) recorded incoming solar irradiance and reflected irradiance during surveys to account for changes in solar intensity. The sunshine sensor module was fitted to the Phantom 3 Professional using a Parrot Sequoia Mount Kit.
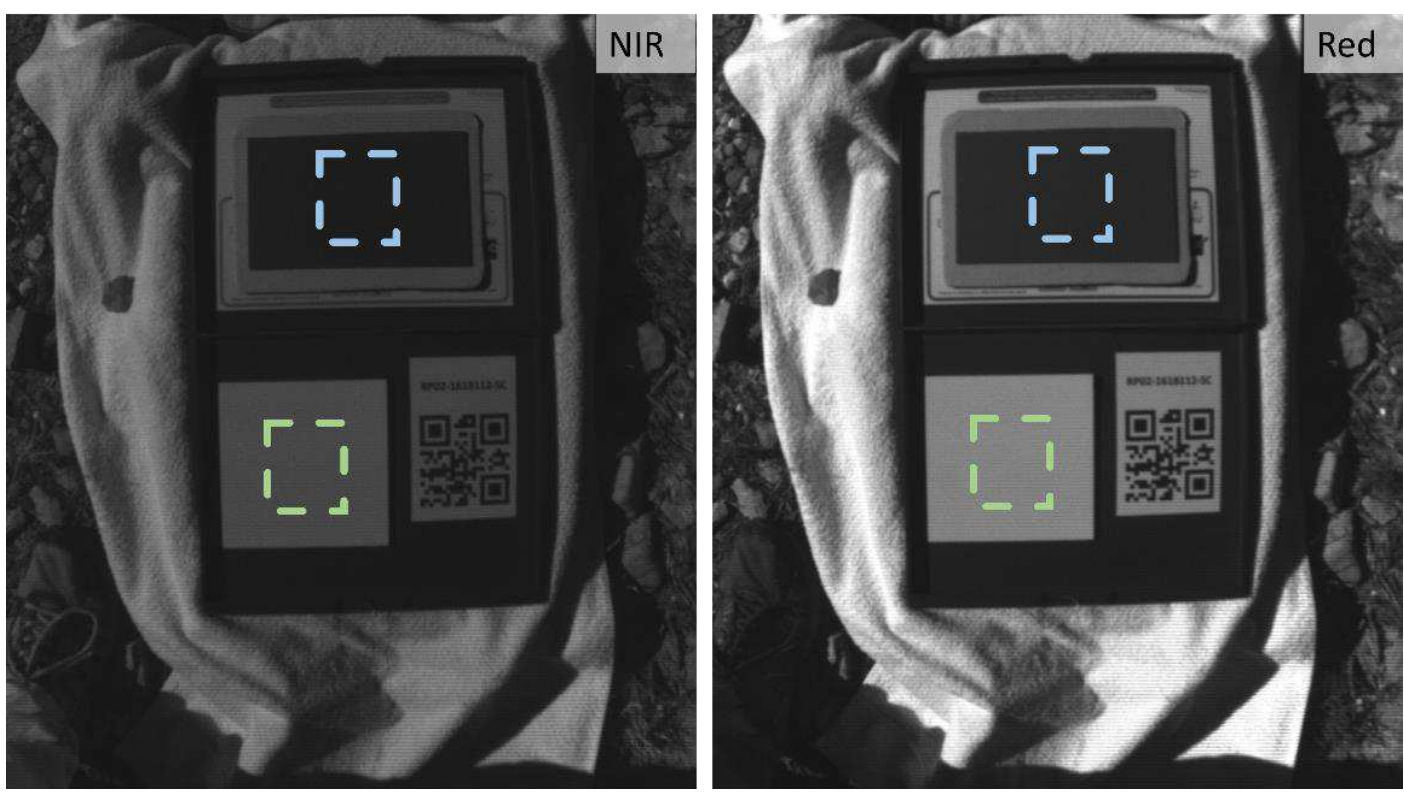

Fig. 1 Radiometric calibration panels including the Micasense calibrated reflectance panel (green squares) and $18 \%$ gray cards (blue squares).

For each survey date, corrected and calibrated red and NIR images were orthorectified and converted into composite single-band orthomosaic images which covered the full area of the sites (Fig. 2). Red and NIR orthomosaic images were automatically aligned by Pix4D software using the known lens offset positions on the camera. Orthomosaic NDVI images were then generated using the equation:

1)

$$
N D V I=\frac{N I R-R E D}{N I R+R E D}
$$

Repeated orthomosaic NDVI layers were produced for Fynbos and Renosterveld sites over 5 separate surveys (i.e. Feb 2017, Oct 2017, Apr 2018, Oct 2018, May 2019) (Fig. 2). In addition to NDVI orthomosaic images, matching RBG orthomosaic images were also generated for comparison at each time step. 


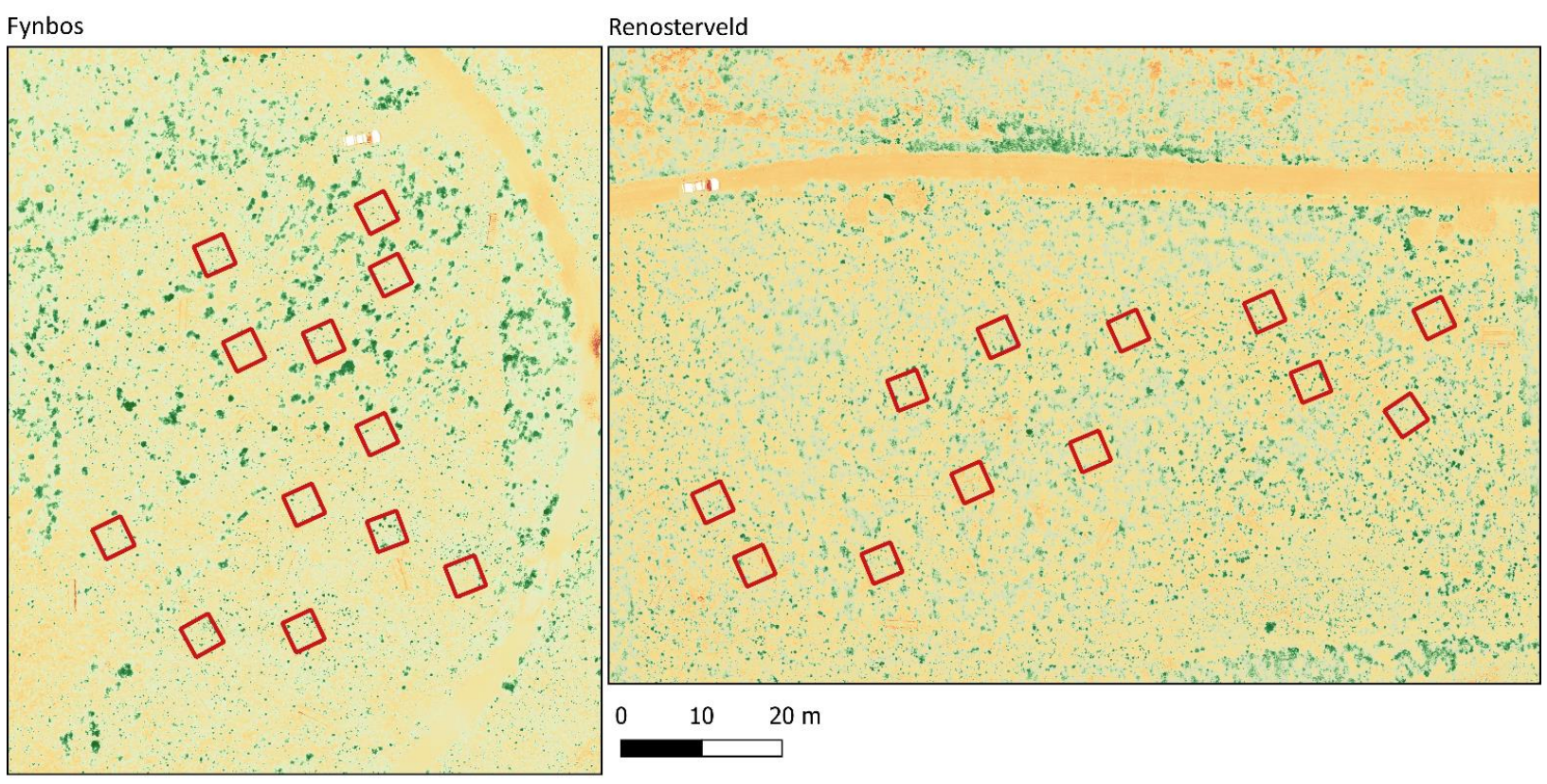

Fig. 2 NDVI orthomosaic layers for post-fire Fynbos and Renosterveld sites from a single time-step. Colour scales represent NDVI -1 (red): 1 (green). Red squares represent $16 \mathrm{~m}^{2}$ focal plots in which ground measurements of vegetation area were taken. Repeated orthomosaic layers were obtained for Nov 2016, Feb 2017, Oct 2017, Apr 2018, Oct 2018, May 2019.

\subsection{Relative radiometric normalization of NDVI orthomosaic images}

\subsubsection{Tracking pseudo-invariant features (PIFs) within NDVI image layers to assess temporal distortion in}

repeated imagery.

While we followed the recommended protocols for the collection and calibration of red and NIR images using the Parrot Sequoia and Pix4DMapper, we found that additional steps were required to obtain temporal radiometric stability in repeated orthomosaic NDVI images. We achieved this by applying additional radiometric corrections directly to NDVI orthomosaic images. The radiometric stability of the NDVI pixel values in repeated orthomosaic image layers was investigated by tracking the variation in pseudo-invariant features (PIFs) within the NDVI image layers over time. PIFs are commonly used for the relative radiometric normalization of time-series data which is often required for accurate land cover detection (Bao et al., 2012; Zhou et al., 2016). Here PIFs were considered to be landscape features or objects within images which were not expected to vary over time in terms of their NDVI value, regardless of factors such as season or time after fire. PIFs were thus intended to reveal variation in the images which was not driven by real landscape changes, but rather by artifacts of the imagery acquisition process. PIFs included a trackable low-value NDVI feature and a 
high-value NDVI feature. PIFs were tracked over time using polygons in fixed positions aligned with the orthoimages. A combination of low and high value PIFs were necessary to monitor image stability over the full spectrum of possible pixel values. This is because temporal radiometric distortion in pixel values over repeated images was not necessarily equal across all pixels and could arise from the compression or expansion of NDVI scale rather than image-wide pixel value shifts. Pixel frequency histograms were used to visually compare image pixel values over time in corrected and uncorrected imagery.

\subsubsection{Selection of pseudo-invariant features}

We selected bare soil patches as low-NDVI-value PIFs (Fig. 3) because we assumed that soil NDVI values should not change over time and should have consistent, low NDVI value. The central pixels of dense, unburnt, off-plot, evergreen shrubs were selected as high value PIFs (Fig. 3). We assumed that these pixels represented $100 \%$ evergreen leaf coverage with no soil pixels included. Using dense, evergreen vegetation polygons as high PIFs could be problematic in some cases seeing that some seasonal variation in leaf NDVI might be expected. Ideally, PIFs should be inert, whereas vegetation NDVI values may vary. However, in this scenario, we argue that the central pixels of dense, evergreen, deep-rooted shrubs were likely to have minimal seasonal photosynthetic variation (van Blerk et al., $2021 \mathrm{~b}$ ) and were thus the best available option for a high value PIF. For each PIF, multiple polygons (>20) were chosen and tracked over time.

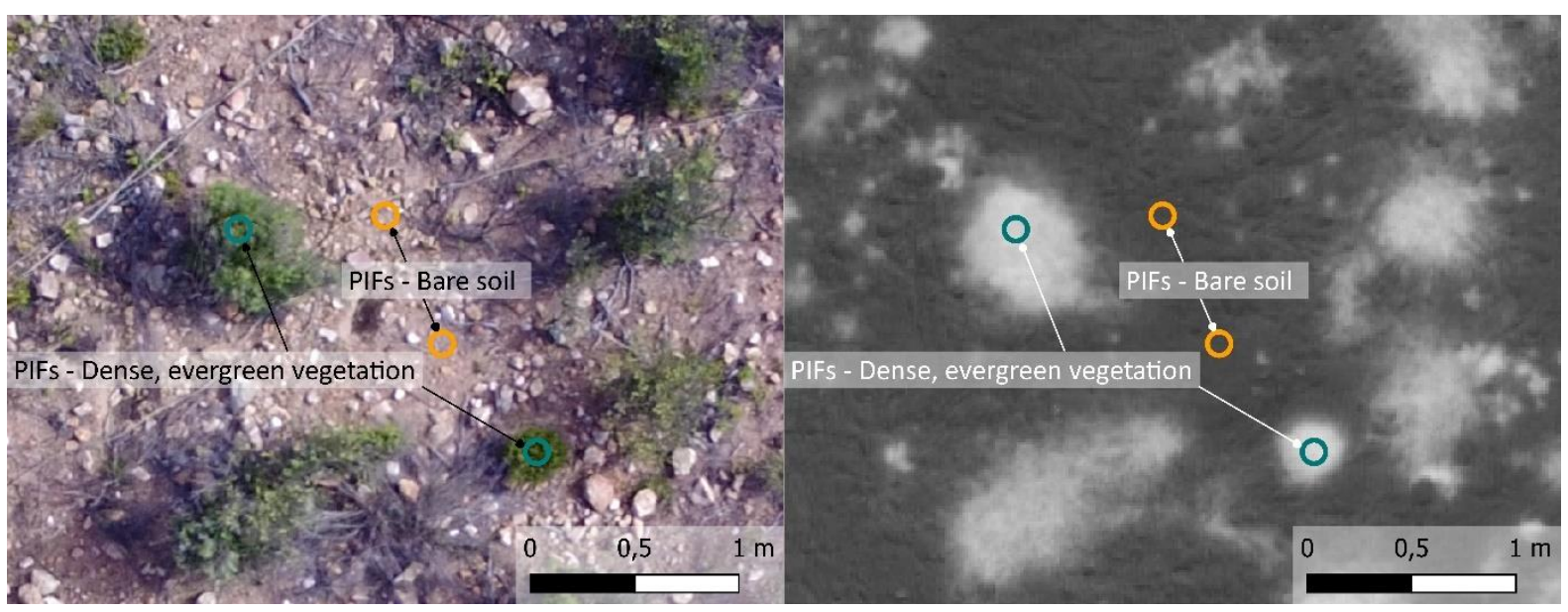

Fig. 3 Example of pseudo-invariant features (PIFs) shown in RGB (left) and greyscale NDVI (right) images. Low NDVI value PIFs = bare soil (orange circles). High-value PIFs = dense, evergreen vegetation (blue circles). 
195 Relative radiometric normalization of imagery was used to adjust the pixel values of image layers relative to the

196 PIFs of the first image in the sequence (i.e. reference image). Pixel adjustment equations were modeled on the

197 linear relationship between the PIF values of uncorrected and reference images (Fig. 4), using the sum of least squares. Pixel values of uncorrected images were modified to their target values according to the formula

$$
\mathrm{y}_{\mathrm{i}}=m x+c
$$

Where $\mathrm{y}=$ the value of a given pixel $i$ within the reference image, $m=$ slope of the relationship between

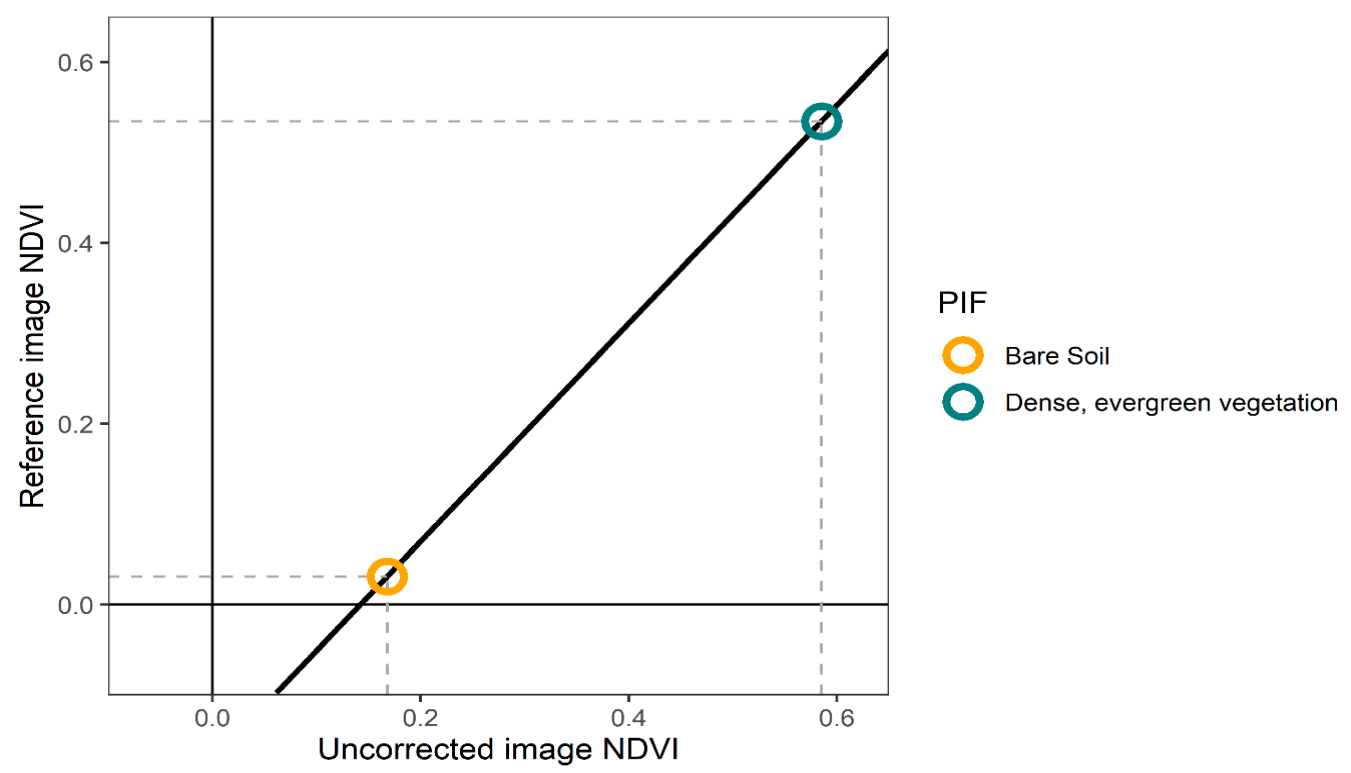

Fig. 4 Diagram showing the relationship between PIFs from an uncorrected image and reference image (i.e. first image in time-series). Dashed grey lines trace the PIF values to their positions on the $\mathrm{x}$ and $\mathrm{y}$ axes. Trendline is displayed in black and represents the line from which the normalization equation is derived. This process was applied to images from each survey date. 
214 We used a combination of QGIS (QGIS Development Team, 2009. QGIS Geographic Information System.

215 Open Source Geospatial Foundation. http://qgis.org) and R (R Core Team 2020. https://www.r-project.org) to 216 extract and summarize pixel values from the multispectral imagery over the 5 survey dates. For both the Fynbos

217 and Renosterveld site, polygons were used to delineate the $16 \mathrm{~m}^{2}$ treatment plots in the series of aligned RGB

218 (Fig. 5 a) and NDVI (Fig. 5 b) orthomosaic layers over time. RGB image layers were used as a guide for 219 choosing the correct positioning of polygons. The bases of the upright steel poles in the corner of each plot 220 served as points of orientation for alignment.

221 The exact_extract() function was used to summarize NDVI pixel values from within plot polygons or fixed PIF 222 polygons.

\subsection{Calculating vegetation area cover using NDVI-thresholding}

224 We used a simple NDVI image thresholding technique to isolate vegetation pixels from the soil pixels within the 225 NDVI orthomosaic images (Fig. 5 c). This entailed removing all pixels from NDVI orthomosaic layers below a 226 chosen NDVI threshold value $<0.2$ (Fig. 5 c). The presence/absence of pixels then corresponded with 227 vegetation cover versus no vegetation cover in images respectively, allowing for the quantification of highresolution area cover per plot (Fig. $\mathbf{5}$ d). The chosen NDVI threshold value was higher than the mean values of soil PIFs and was visually adjusted in QGIS until it most successfully corresponded to vegetation patches. Area cover was measured by multiplying the number of pixels above the threshold of 0.2 NDVI by the squared pixel area. Overlaying the threshold images onto RGB images allowed for a visual comparison of threshold area and visible vegetation patches (Fig. 5 e,). Because NDVI pixel density histograms were unimodal, we did not attempt to use automatic thresholding algorithms (e.g. Otsu, 1979). Instead, we chose to manually select and adjust thresholds based on available pixel information. We also considered automatic edge detection methods (e.g. level setting) to calculate the area of vegetation within NDVI images (e.g. Ghazal et al., 2015) but due to the computational complexities of such a method, we settled with our simpler, more accessible approach. 


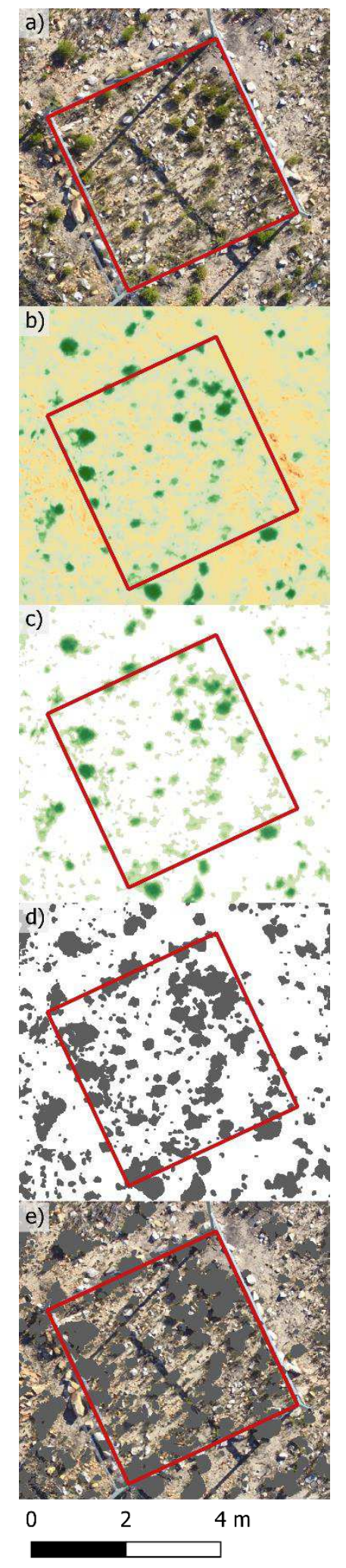

Fig. 5 Process of extracting NDVI-derived area cover data from images. a) RGB orthomosaic showing vegetation and soil. Red squares represent $16 \mathrm{~m}^{2}$ focal plots. b) NDVI orthomosaic -1 (red): 1 (green) c) Vegetation-specific NDVI using a threshold of NDVI > 0.2 d) Area cover polygons derived from vegetation specific NDVI e) Overlay of vegetation area-cover polygons on RGB images for visual comparison. 
244 Detailed plant demographic surveys were conducted at the same time as UAV-based surveys. Demographic

245 surveys included counting and measuring the area and height of every plant within each of the $12,16 \mathrm{~m}^{2}$ focal

246 plots. Over 60000 individual measurements were made over the duration of the experiment (van Blerk et al., 2472021 a).

248 Linear relationships between UAV-based area measurements and ground area measurements were compared

249 between uncorrected and corrected imagery. R-squared values were calculated using the sum of least squares.

250

251

252

253

254

255

256

257

258

259

260

261

262

263

264

265 
269 Bare soil PIF polygons clearly showed elevated NDVI values in Fynbos during October 2018 (Fig. 6 c) and

270 corresponded with a large increase in mean plot NDVI (Fig. 6 a). Soil pixel values had a strong overall

271 influence on mean plot NDVI because the majority of image pixels represented soil in the post-fire environment.

272 Renosterveld soil PIF polygons also displayed slightly reduced NDVI values in April 2018 and May 2019 (Fig.

2736 d), contributing to lower mean plot NDVI values during those months (Fig. 6 b). In both Fynbos and

274 Renosterveld, shifts in soil PIF polygons did not correspond with equivalent shifts in dense, evergreen

275 vegetation polygons (Fig. 6 e, f). This indicated that the nature of the image radiometric distortion was non-

276 linear and was not an equal shift in pixel values across the full range of NDVI values. Higher NDVI values were

277 relatively unaffected by contrast problems which so clearly affected low NDVI values.

278 Image pixel density histograms (Fig. 7) provided a visual confirmation that compression or expansion of

279 histogram peaks had occurred in some layers (e.g. October 2018 Fynbos) rather than range shifts which would have shown equal shifts in all pixels.

281

282

283

284

285

286 
a)

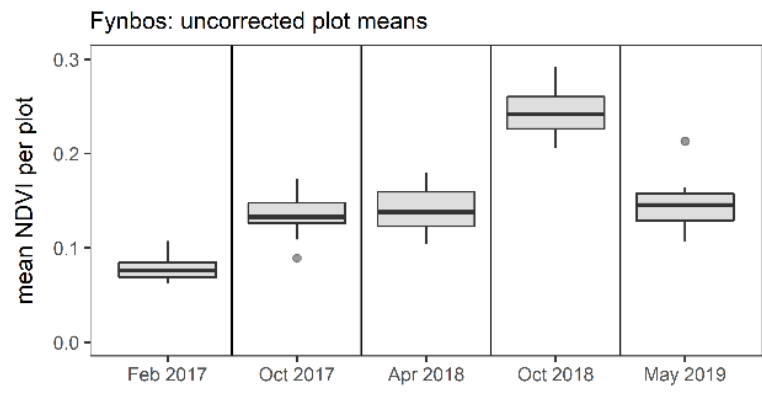

c)

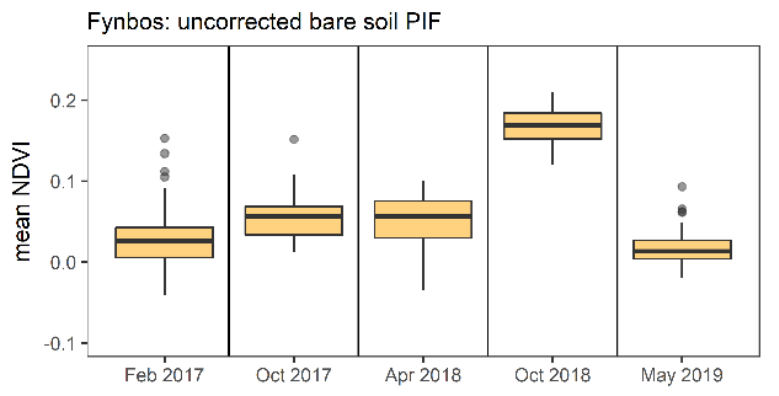

e)

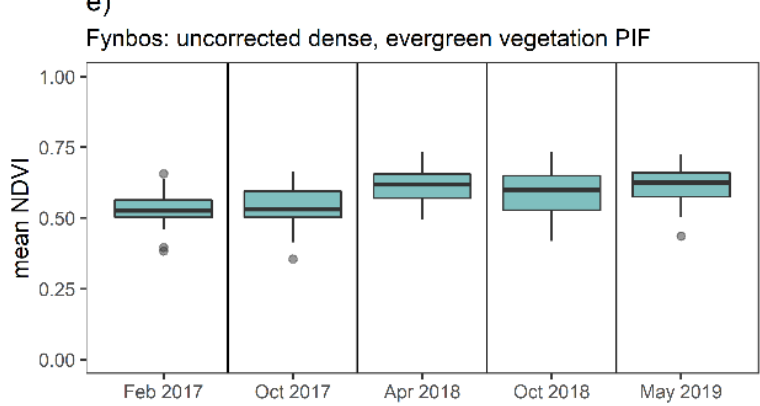

b)

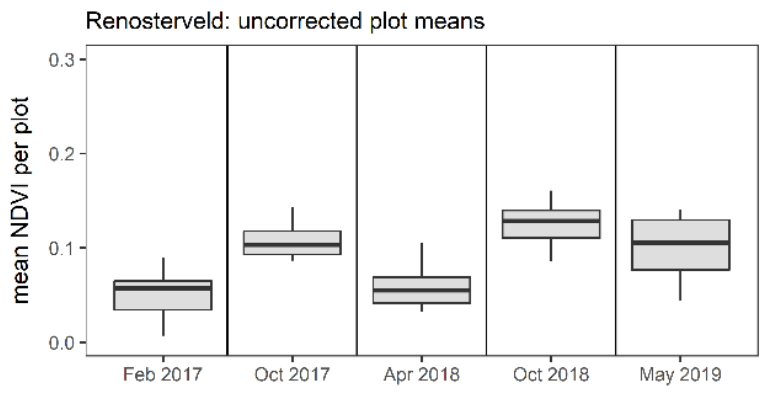

d)

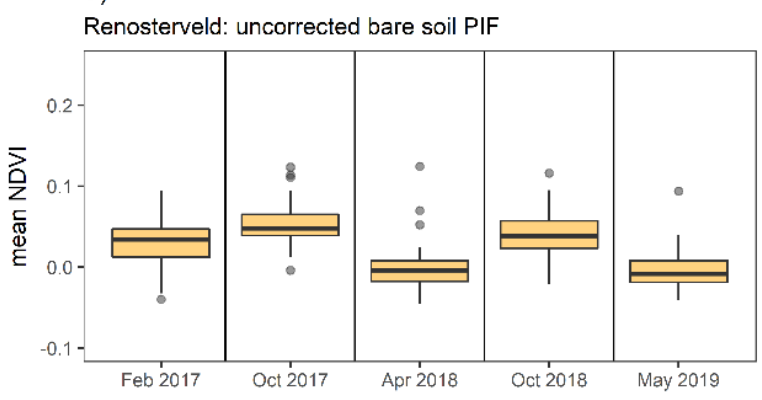

f)

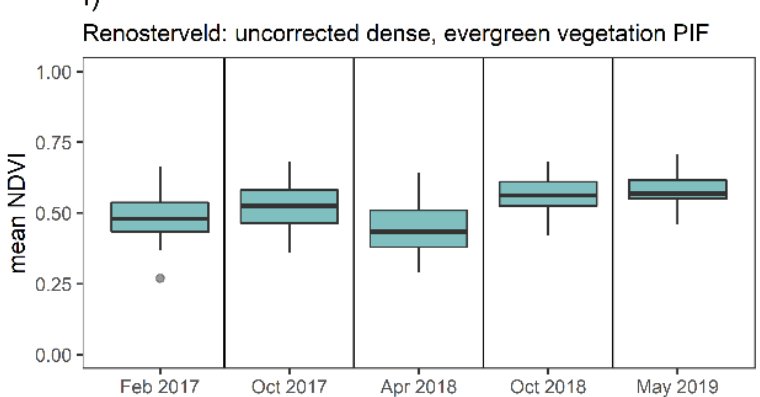

Fig. 6 a, b) Mean NDVI per plot using uncorrected Fynbos and Renosterveld orthomosaic NDVI images. c, d) mean NDVI of bare soil PIFs (pseudo invariant feature) and e, f) mean NDVI of dense, evergreen vegetation PIFs for uncorrected image layers.

\subsection{Identifying possible software and hardware issues}

Image radiometric distortion over time, represented by non-matching histogram peaks (Fig. 7), was not introduced by any of the Pix4D image processing corrections applied during the production of the NDVI orthomosaic images. This was tested by re-processing imagery using different combinations of software corrections available. These corrections included i) camera corrections (e.g. vignetting, ISO, dark current etc.), ii) radiometric calibration panel corrections, iii) sunshine sensor corrections. Camera corrections dramatically improved the similarity of histograms (shape and range) over time relative to the raw orthomosaic images. 
panel versus 18\% grey panel) in Pix4D had little effect on the output data suggesting that the panels were reliable reflectance references. Additionally, applying image corrections using radiometric calibration panels and/or the sunshine sensor resulted in equivalent output image pixel density histograms, indicating redundancy between radiometric calibration using field panels and the sunshine sensor, as suggested by the developer.

Overall, these tests indicated that image distortion in some layers was not directly a result of software processing through Pix4D or calibration panel issues. Rather, this finding points to deeper issues associated with the functioning of the camera or survey conditions during some UAV flights including camera angle, camera temperature, convective warm air, atmospheric moisture and sun angle.

Notably, we observed that the UAV would briefly tilt when taking photographs during some surveys. This could potentially lead to significant changes in the angle of the camera relative to the ground. If this was the cause of image distortion in some layers, then the clear contrast between the distortion effects on pixels with low NDVI values (i.e. soil PIFs) versus those with high values (i.e. dense, evergreen vegetation PIFs) could be explained by differences in the sensitivity of object reflectivity to slight differences in camera/sun angle during the surveys. For example, the complex, diffuse reflectance of fine-leaved vegetation is not likely to vary strongly with variation in measurement angles, suggesting that slight changes in sun or camera angle had relatively little effect on the reflectance values captured by the camera. Conversely, angular or flat surfaces, such as soil and rock, could be more susceptible to such angle-related factors. Additionally, other complex, angle-related phenomena, such as the bidirectional reflectance effect, could differentially alter the reflectance characteristics of red and NIR wavelengths based on sun and sensor angles thus leading to complex image distortion (Hapke, 1981). While we endeavoured to uncover the exact cause of image distortion, the cryptic nature of this distortion proved to be too complex to identify with the information available. Therefore, we felt that post-processing image correction (i.e. direct correction on NDVI layers) would be the most robust and practical solution.

\subsection{Image normalisation based on PIFs}

Relative radiometric normalisation resulted in matching PIFs over repeated images in both sites (Fig. 7). This had the effect of closely aligning the peaks of pixel frequency histograms over time (Fig. 7). Uncorrected image histograms were relatively similar to corrected image histograms at most time-periods in both sites with the exception of the Fynbos site in October 2018 where a large transformation effect is clearly visible (Fig. 7). It was apparent that matching dense, evergreen vegetation PIFs had a relatively small effect on the shape of histograms compared to the effect of matching bare soil PIFs, which strongly shifted histogram peaks. 
329 Importantly, even after matching dense, evergreen vegetation PIFs over time, the density of the histograms

330 toward upper NDVI values was still able to increase over time, indicating an increase in the fraction of

331 vegetation relative to bare soil. In corrected imagery, plot NDVI patterns (Fig. 7) did not include the large,

332 spurious increases in mean plot NDVI which were previously related to noise in the bare soil PIFs in

333 uncorrected imagery (Fig. 6).

334

335

336

337

338

339

340 
Fynbos

Feb 2017

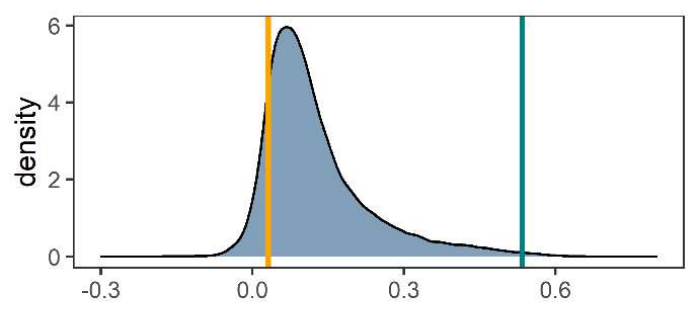

Oct 2017

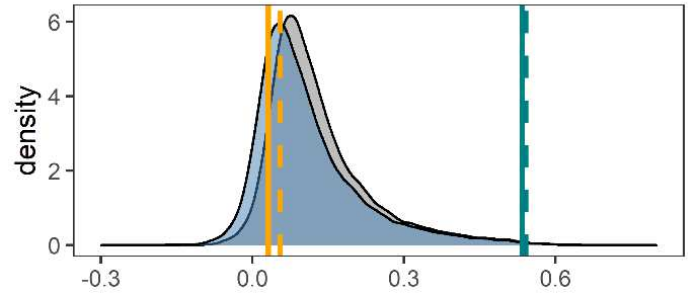

Apr 2018

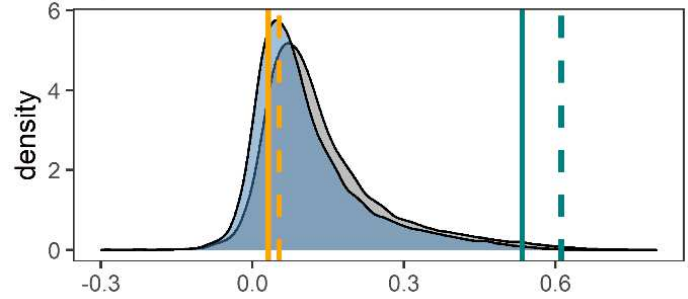

Oct 2018

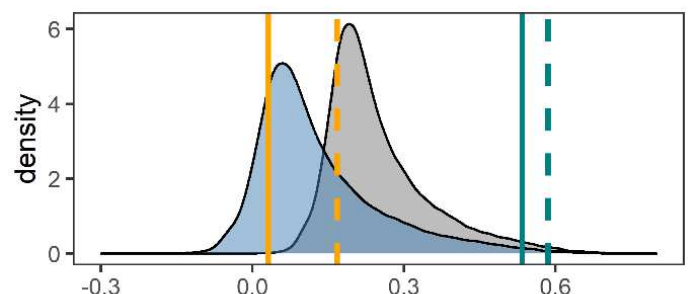

May 2019

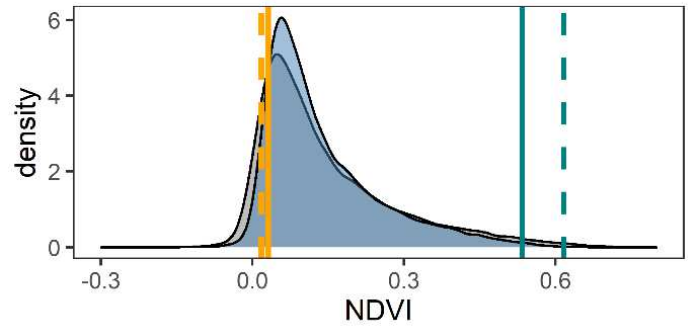

Renosterveld

Feb 2017

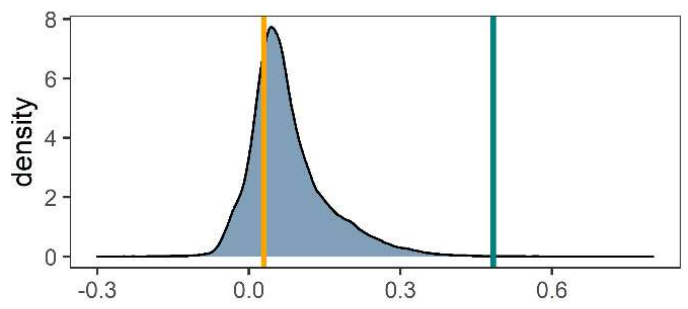

Oct 2017

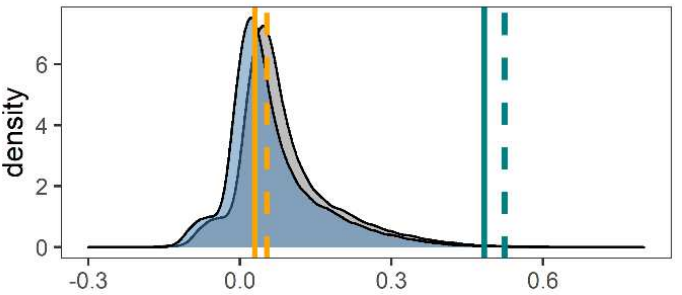

Apr 2018

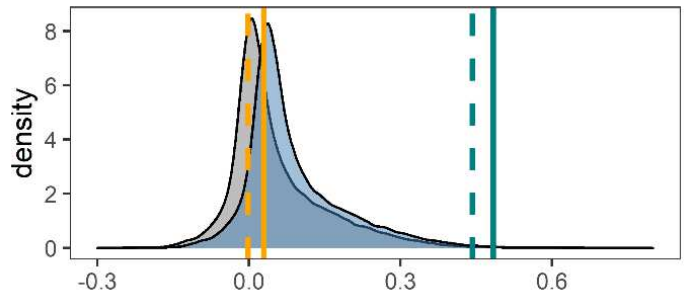

Oct 2018

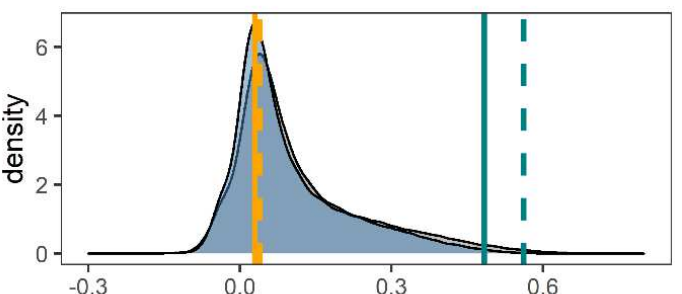

May 2019

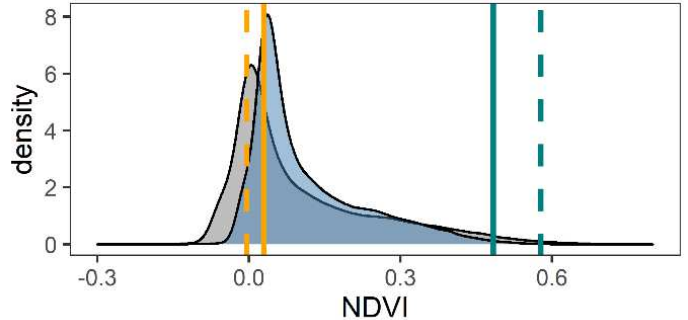

Fig. 7 NDVI pixel density histograms for uncorrected (grey) and corrected (blue) image layers. Solid vertical lines represent corrected PIF values for bare soil (yellow) and dense, evergreen vegetation (blue). Dashed vertical lines represent uncorrected PIF values. 
a)

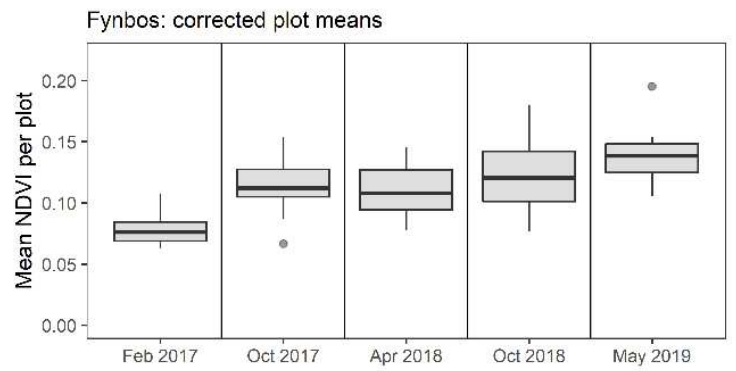

c)

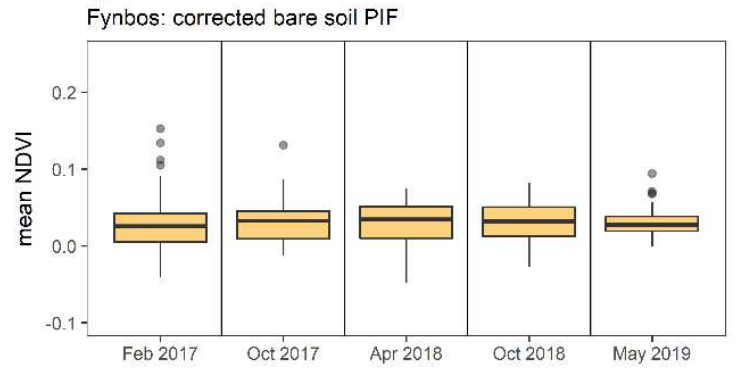

e)

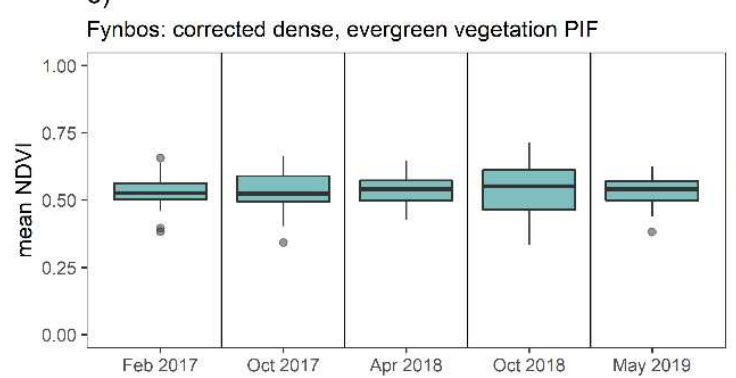

b)

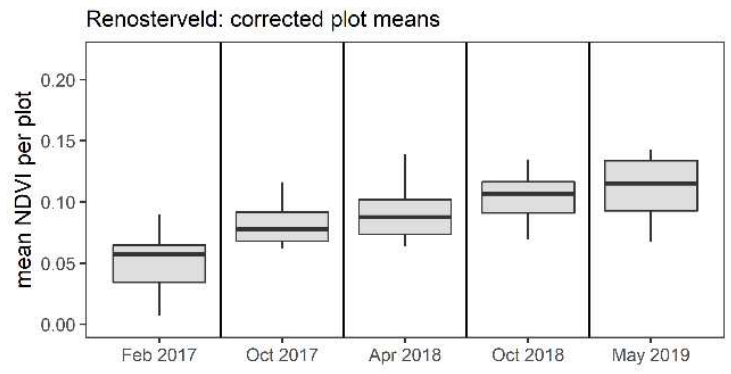

d)

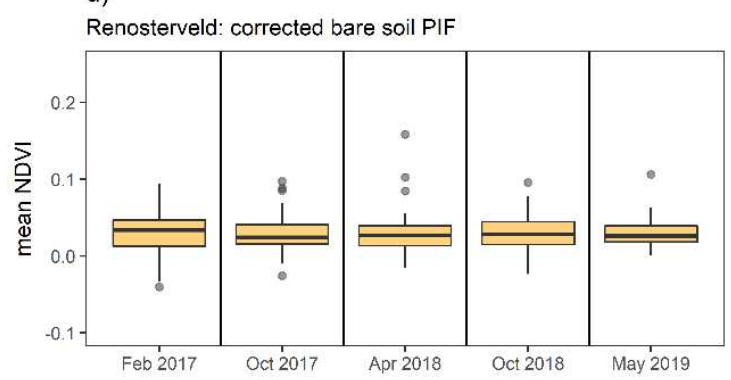

f)

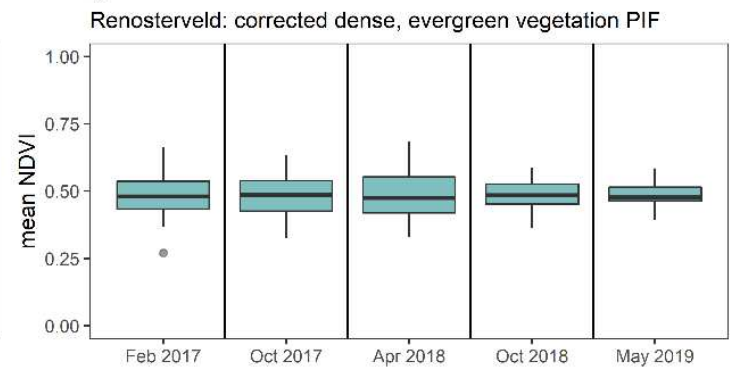

Fig. 8 a, b) Mean NDVI per $16 \mathrm{~m}^{2}$ plot using corrected Fynbos and Renosterveld orthomosaic NDVI images. c, d) mean NDVI of bare soil PIFs (pseudo invariant feature) and e, f) mean NDVI of dense, evergreen vegetation PIFs for corrected image layers.

\subsection{Using NDVI thresholding to measure vegetation cover}

351 A threshold of NDVI $<0.2$ was found to accurately isolate vegetation patches from the environmental features

352 (i.e. rock and soil) (Fig. 9). This was confirmed visually using the RGB images. However, consistently isolating vegetation using a common threshold was problematic using uncorrected imagery if pixel distortion issues were present in some layers, such as in the uncorrected Fynbos layers in October 2018). Image distortion led to soil values being included above the chosen NDVI threshold (Fig. 9).

In the case of the Fynbos Oct2018 layer, this was explained by the compression of low values toward high values which was clearly visible in the frequency histogram (Fig. 7, 8). Inclusion of soil pixels above the vegetation threshold had obvious negative implications for area estimates and vegetation specific signal 


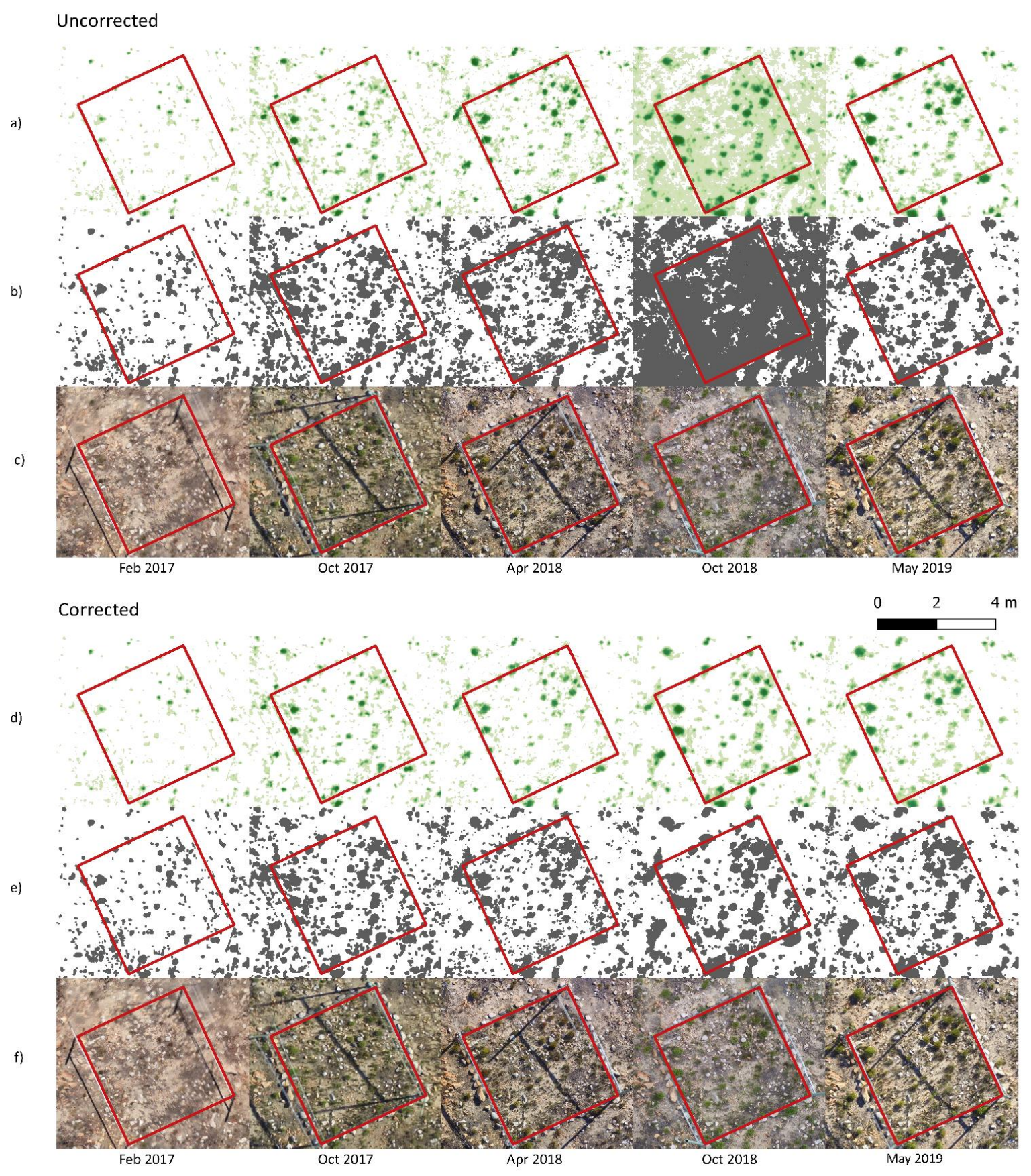

Fig. 9 a, d) Post-fire vegetation-specific NDVI (NDVI > 0.2) changes within a $16 \mathrm{~m}^{2}$ focal plot over time. 
369 Relative radiometric normalisation of imagery significantly improved the correlations between UAV-based 370 vegetation cover measurements and corresponding ground-based measurements (Fig. 10) within $16 \mathrm{~m}^{2}$ focal 371 plots. The effect of normalisation was most evident at the Fynbos site where the uncorrected images had a high 372 degree of temporal inconsistency (e.g. October 2018 orthomosaic). At the plot-level, there was substantial 373 variation between UAV-based and ground-based vegetation cover measurements. However, this was likely to be 374 the result of variation within ground-based measurements instead of inconsistencies in UAV-based 375 measurements. Ground-based area measurements did not account for complex canopy shapes of plants and 376 overlap within plots. When summarised at the site level, relative radiometric normalisation dramatically 377 improved correlations between UAV and ground-based area measurements and resulted in strong relationships 378 (i.e. $\mathrm{R}^{2}>0.87$ ). This was particularly relevant in the Fynbos site where UAV-based and ground-based area 379 measurements were not significantly correlated when using uncorrected imagery. 


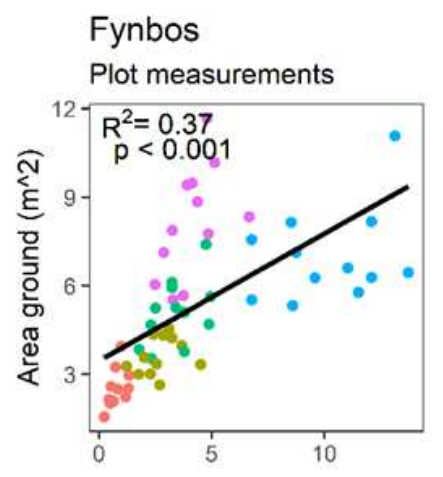

\section{Fynbos}

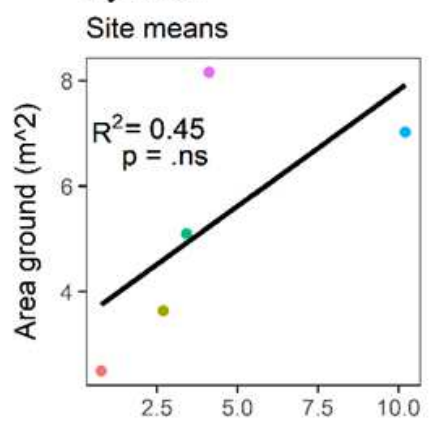

\section{Renosterveld}

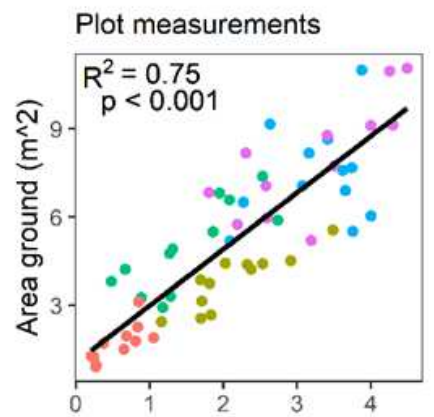

Renosterveld

Site means

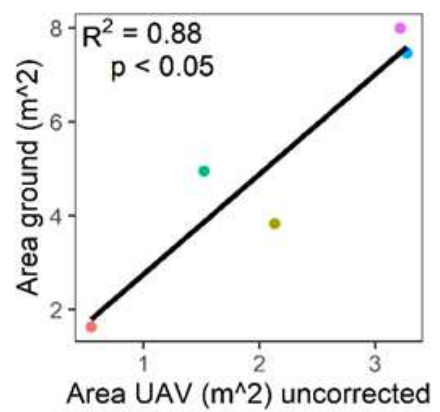

\section{Fynbos}

Plot measurements

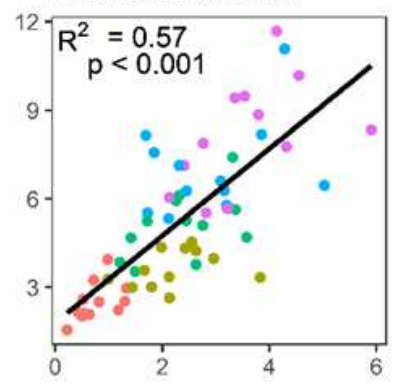

Fynbos

Site means

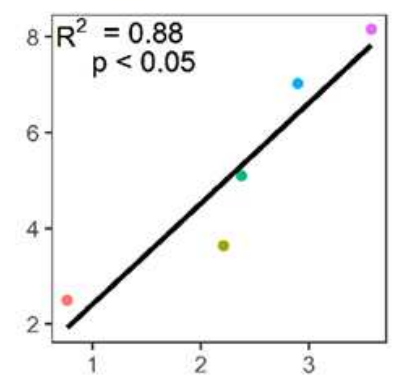

Renosterveld

Plot measurements

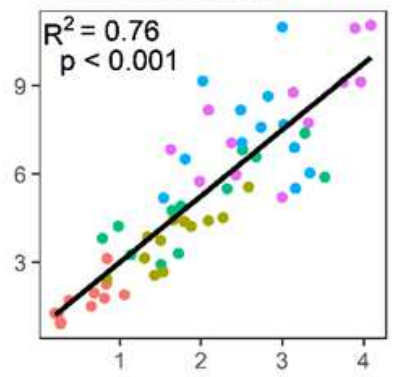

Renosterveld

Site means

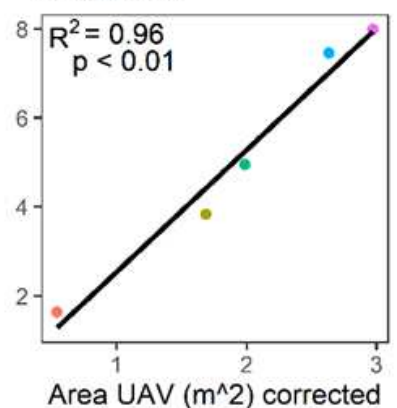

Fig. 10 Comparison of vegetation area cover $\left(\mathrm{m}^{2}\right)$ per $16 \mathrm{~m}^{2}$ focal plot, obtained from ground-based measurements ('Area ground') and UAV-based measurements ('Area UAV'). UAV-based vegetation cover measurements made using uncorrected (left column) and corrected (right column) images are displayed side-byside. Plot measurements include the measured vegetation area cover of each focal plot. Site means represent the mean of plot area cover measurements for each date. 
Our study demonstrated that UAV-mounted multispectral cameras can be a powerful addition to the ecologists' toolbox as it enables repeatable monitoring of high-resolution (i.e. sub-meter) vegetation cover changes over larger spatial scales. However, we caution against adopting a "black-box" approach when using UAV and image processing technology which can result in spurious patterns in data. Our protocol used pseudoinvariant features (PIFs) to assess the radiometric consistency in repeated NDVI images after software calibrations. We then applied appropriate normalisations to images based on PIFs and utilised a simple thresholding method for rapid measurement of vegetation area cover. Extensive ground-truthing revealed that our procedure improved the accuracy of UAV-based vegetation cover measurements.

Default software calibrations in Pix4D (i.e. camera corrections, sunshine sensor and radiometric calibration panels) fell short of producing radiometrically stable NDVI pixel values over time for analysis. This was not necessarily a fault of the software but was rather related to a tendency for uncontrolled environmental, sensor or user error to easily be introduced into repeated surveys (Teillet 1986; Hall et al., 1991). The key challenges to overcome included the identification and correction of image radiometric distortion over the full time-series sequence. Image radiometric distortion can introduce spurious patterns into time-series data, thus limiting the ability to correctly describe vegetation patterns over time (Teillet, 1986; Hall et al., 1991; Zhou et al., 2016). This was clear in the Fynbos image-layers where values representing natural seasonal changes in vegetation were strongly skewed by a single distorted image in October 2018. The effect of image-distortion could also be more subtle and lead to exaggerated vegetation patterns in the data (e.g. Renosterveld April 2018 and May 2019). NDVI orthomosaic layers and can be successful using a minimum of two pseudo-invariant features in the landscape, as long as their values cover a wide enough range of pixel values on the response variable scale (e.g. low and high NDVI). Through the identification of PIFs, the presence and nature of image distortion is revealed, allowing for an appropriate correction to be applied to images. Unavoidably, applying relative radiometric normalization to image pixel values based on dense, evergreen vegetation PIFs in reference images may reduce some of the natural temporal variation in upper NDVI values, associated with leaf-level NDVI seasonality.

414 However, seeing that there was no alternate method to assess the quality of upper NDVI pixel values, we felt that applying an equal normalization across all images was the fairest treatment of the data. Furthermore, 
416 normalization of upper pixel values by matching PIFs to the first image of the time-series did not hinder changes

417 in the shape of density histograms over time. For example, increases in histogram density toward higher NDVI

418 values was apparent over time in corrected imagery and represented increases in vegetation cover.

419 Normalization of upper NDVI pixel values was intended to reduce the chance of Type 1 errors occurring (i.e.

420 detecting vegetation changes that do not exist) (Dekking et al., 2005). While the bias towards avoiding Type 1

421 errors has been identified amongst scientists (Anderegg et al., 2014), we purposefully avoided Type 1 errors

422 because it was apparent how easily they could be introduced into data even by slight image distortion. Notably,

423 image normalization based on features within images was superior to normalization based on histograms

424 themselves. Histogram matching was not appropriate because it results in near-identical histograms over time,

425 thus muting any vegetation change signals within images.

426 While we show that natural landscape features can be used successfully to conduct relative radiometric

427 normalization, this possibility is strongly dependent on the features of the specific environment being surveyed

428 (e.g. evergreen shrubs, stable soils). If no stable soil value or vegetation value exists in the landscape, our

429 proposed methodology would not be applicable. Therefore, including permanent ground control points would

430 ideally replace the organic (bare soil and dense, evergreen vegetation) PIFs used in our methodology. We also

431 recommend including RGB imagery in UAV-based surveys. The ability to visually verify that vegetation indices

432 and area cover polygons correspond with the actual patterns of vegetation cover on the ground is invaluable.

\section{Recommendations}

434 We recommend adopting a protocol to identify and correct temporal radiometric noise in repeated imagery data

435 before extracting ecological or environmental information from imagery (Fig. 11). This should be carried out

436 even if imagery processing software includes calibration and normalisation procedures. Additionally, we

437 recommend testing the quality of UAV-based data by including some degree of ground-truthing. Together, these

438 steps can ensure the production of reliable time-series data. 


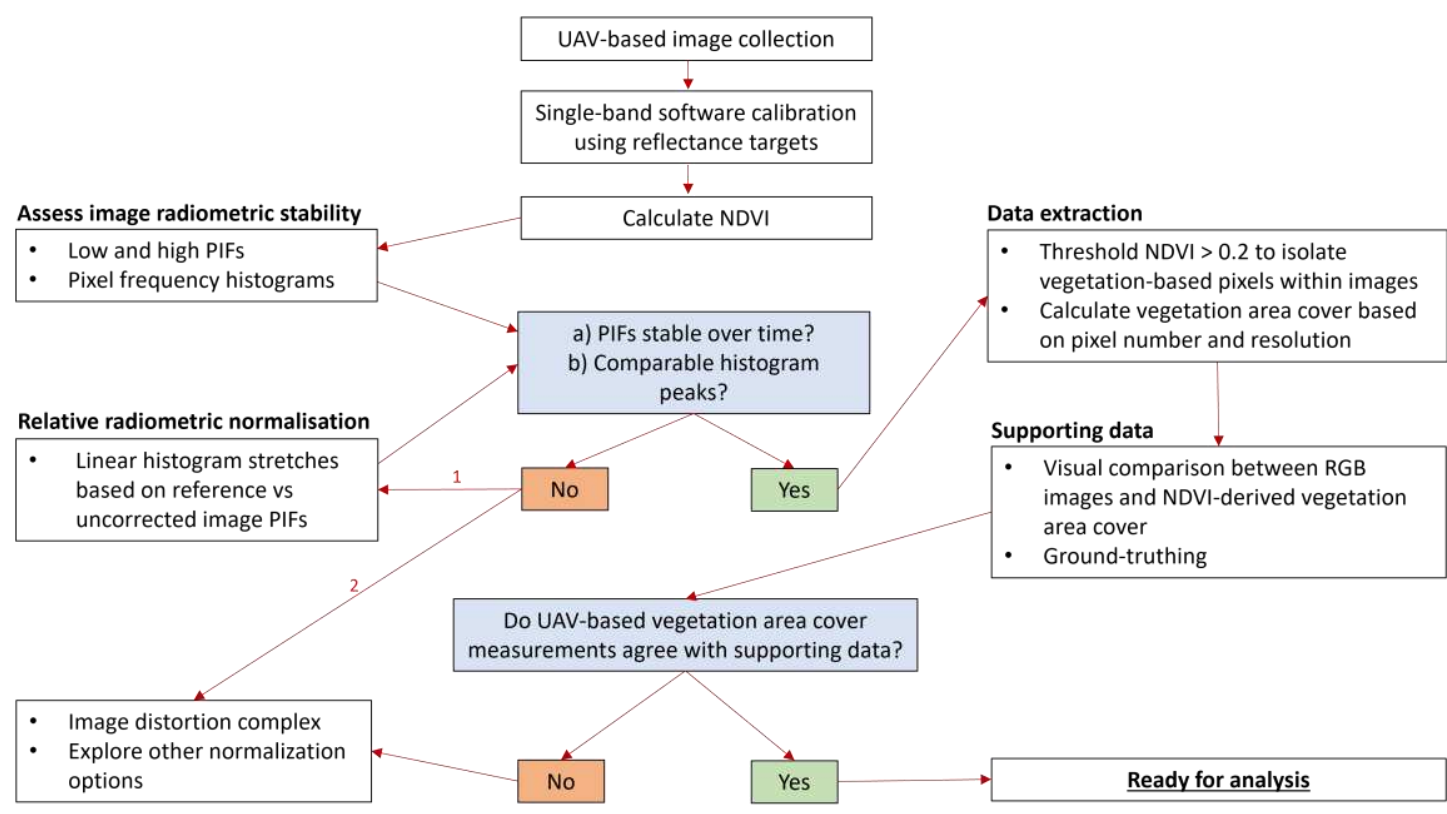

440 Fig. 11 Protocol for UAV-based vegetation cover change monitoring.

441 Overall, if used with caution, UAV-mounted multispectral cameras greatly improve the potential for monitoring

442 fine-scale vegetation changes (sub-meter resolution) across intermediate special and temporal scales. This could

443 lead to a better understanding of how vegetation processes relate to patterns observed at larger spatial scales. 
Anderegg, W. R., Callaway, E. S., Boykoff, M. T., Yohe, G., \& Root, T. Y. L. (2014). Awareness of both type 1 and 2 errors in climate science and assessment. Bulletin of the American Meteorological Society, 95(9), $1445-1451$.

Aplin, P. (2005). Remote sensing: ecology. Progress in Physical Geography, 29(1), 104-113.

Bao, N., Lechner, A. M., Fletcher, A., Mulligan, D., Mellor, A., \& Bai, Z. (2012). Comparison of relative radiometric normalization methods using pseudo-invariant features for change detection studies in rural and urban landscapes. Journal of Applied Remote Sensing, 6(1), 063578.

Bergstedt, J., Westerberg, L., \& Milberg, P. (2009). In the eye of the beholder: bias and stochastic variation in cover estimates. Plant ecology, 204(2), 271-283.

Cammeraat, E., van Beek, R., \& Kooijman, A. (2005). Vegetation succession and its consequences for slope stability in SE Spain. Plant and Soil, 278(1), 135-147.

Cramer, M. D., \& Hoffman, M. T. (2015). The consequences of precipitation seasonality for Mediterraneanecosystem vegetation of South Africa. PloS one, 10(12), e0144512.

Dekking, F. M., Kraaikamp, C., Lopuhaä, H. P., \& Meester, L. E. (2005). A Modern Introduction to Probability and Statistics: Understanding why and how. Springer Science \& Business Media.

Duveiller, G., \& Defourny, P. (2010). A conceptual framework to define the spatial resolution requirements for agricultural monitoring using remote sensing. Remote Sensing of Environment, 114(11), 2637-2650.

Esler KJ, Von Staden L, Midgley GF. 2015. Determinants of the Fynbos/ Succulent Karoo biome boundary: insights from a reciprocal transplant experiment. South African Journal of Botany 101: 120-128.

Estrany, J., Ruiz, M., Calsamiglia, A., Carriquí, M., García-Comendador, J., Nadal, M., ... \& Gago, J. (2019). Sediment connectivity linked to vegetation using UAVs: High-resolution imagery for ecosystem management. Science of the Total Environment, 671, 1192-1205.

Ghazal, M., Al Khalil, Y., \& Hajjdiab, H. (2015, December). UAV-based remote sensing for vegetation cover estimation using NDVI imagery and level sets method. In 2015 IEEE International Symposium on Signal Processing and Information Technology (ISSPIT) (pp. 332-337). IEEE. 
Gitas, I., Mitri, G., Veraverbeke, S., \& Polychronaki, A. (2012). Advances in remote sensing of post-fire vegetation recovery monitoring - a review. Remote sensing of biomass-Principles and applications, 1 , 334.

Hall, F. G., Strebel, D. E., Nickeson, J. E., \& Goetz, S. J. (1991). Radiometric rectification: toward a common radiometric response among multidate, multisensor images. Remote sensing of environment, 35(1), 1127.

Hapke, B. (1981). Bidirectional reflectance spectroscopy: 1. Theory. Journal of Geophysical Research: Solid Earth, 86(B4), 3039-3054.

Marcial-Pablo, M. D. J., Gonzalez-Sanchez, A., Jimenez-Jimenez, S. I., Ontiveros-Capurata, R. E., \& OjedaBustamante, W. (2019). Estimation of vegetation fraction using RGB and multispectral images from UAV. International journal of remote sensing, 40(2), 420-438.

Mucina, L., \& Rutherford, M. C. (2006). The vegetation of South Africa, Lesotho and Swaziland. South African National Biodiversity Institute.

Otsu, N. (1979). A threshold selection method from gray-level histograms. IEEE transactions on systems, man, and cybernetics, 9(1), 62-66.

Port, U., Brovkin, V., \& Claussen, M. (2012). The influence of vegetation dynamics on anthropogenic climate change. Earth System Dynamics, 3(2), 233-243.

Rasmussen, J., Ntakos, G., Nielsen, J., Svensgaard, J., Poulsen, R. N., \& Christensen, S. (2016). Are vegetation indices derived from consumer-grade cameras mounted on UAVs sufficiently reliable for assessing experimental plots?. European Journal of Agronomy, 74, 75-92.

Shoshany, M., Lavee, H., \& Kutiel, P. (1995). Seasonal vegetation cover changes as indicators of soil types along a climatological gradient: a mutual study of environmental patterns and controls using remote sensing. International Journal of Remote Sensing, 16(12), 2137-2151.

Sotille, M. E., Bremer, U. F., Vieira, G., Velho, L. F., Petsch, C., \& Simões, J. C. (2020). Evaluation of UAV and satellite-derived NDVI to map maritime Antarctic vegetation. Applied Geography, 125, 102322. 

Sensing, 166, 15-25.

508

Teillet, P. M. (1986). Image correction for radiometric effects in remote sensing. International Journal of Remote Sensing, 7(12), 1637-1651.

Tucker, C. J. (1979). Red and photographic infrared linear combinations for monitoring vegetation. Remote sensing of Environment, 8(2), 127-150.

van Blerk, J. J., West, A. G., Altwegg, R., \& Hoffman, M. T. (2021) a. Does a trade-off between growth plasticity and resource conservatism mediate post-fire shrubland responses to rainfall seasonality?. New Phytologist, 230(4), 1407-1420.

van Blerk, J. J., West, A. G., Altwegg, R., \& Hoffman, M. T. (2021) b. Post-fire summer rainfall differentially affects reseeder and resprouter population recovery in fire-prone shrublands of South Africa. Science of The Total Environment, 788, 147699.

van Iersel, W., Straatsma, M., Addink, E., \& Middelkoop, H. (2018). Monitoring height and greenness of nonwoody floodplain vegetation with UAV time series. ISPRS journal of photogrammetry and remote sensing, 141, 112-123.

Veraverbeke, S., Gitas, I., Katagis, T., Polychronaki, A., Somers, B., \& Goossens, R. (2012). Assessing post-fire vegetation recovery using red-near infrared vegetation indices: Accounting for background and vegetation variability. ISPRS Journal of Photogrammetry and Remote Sensing, 68, 28-39.

West, A. G., Dawson, T. E., February, E. C., Midgley, G. F., Bond, W. J., \& Aston, T. L. (2012). Diverse functional responses to drought in a Mediterranean-type shrubland in South Africa. New Phytologist, 195(2), 396-407.

Zhang, C., \& Kovacs, J. M. (2012). The application of small unmanned aerial systems for precision agriculture: a review. Precision agriculture, 13(6), 693-712.

Zhou, H., Liu, S., He, J., Wen, Q., Song, L., \& Ma, Y. (2016). A new model for the automatic relative radiometric normalization of multiple images with pseudo-invariant features. International Journal of Remote Sensing, 37(19), 4554-4573. 
532 Websites cited:

533 https://www.dji.com

534 https://www.parrot.com

535 https://micasense.com

536 http://qgis.org

537 https://www.r-project.org

538

539 Acknowledgements

540 Funding was generously provided by the NRF (93380 to AGW and 119125 to RA) and ACCESS groups

541 (114696). Thank you to the Plant Conservation Unit for their contribution to the establishment of the Drie

542 Kuilen rainfall manipulation experiment. JJvB was supported by a UCT Science Faculty Scholarship. We are

543 grateful to the NCC and the Drie Kuilen Nature Reserve for providing access and support. Thank you to the

544 many field assistants who were eager to help throughout the research process.

\section{Author contributions}

$546 \mathrm{JJvB}, \mathrm{AGW}, \mathrm{MTH}, \mathrm{RA}$ and JS conceptualized the research. JJvB, MTH and AGW collected the data. JJvB

547 processed the data and wrote the manuscript, with contributions from AGW, MTH, RA and JS.

\section{Data availability}

549 The data used during the current study are available from the corresponding author on reasonable request.

\section{Author declarations}

551 The authors have no relevant financial or non-financial interests to disclose. 